\title{
ENFERMEDAD DE FABRY
}

\author{
FABRY DISEASE
}

Martín Gómez-Luján'

\begin{abstract}
RESUMEN
La enfermedad de Fabry es una enfermedad de depósito lisosomal causada por la deficiencia de la enzima alfa galactosidasa $\mathrm{A}$, con patrón de herencia ligado al cromosoma $X$. El cuadro clínico tiene una variedad de síntomas y signos; y se han descrito una variante clínica clásica y variantes clínica no clásica como la renal o cardíaca. El diagnóstico es clínico y de laboratorio e incluso molecular y en algunos casos se necesita histología del órgano comprometido. El tratamiento en los últimos años sigue siendo sintomático y con terapia de reemplazo enzimático; aunque ya se cuenta con chaperonas para el tratamiento de un grupo de pacientes con mutaciones "susceptibles" y están en estudio la terapia génica y la nanotecnología. Se debe tener un abordaje multidisciplinario, dada la variedad de síntomas y signos en su presentación. La detección temprana y el inicio temprano de la terapia podrían mejorar la calidad de vida y limitar la progresión de la enfermedad; y finalmente siempre debe considerarse la consejería genética.
\end{abstract}

Palabras clave: Enfermedad de Fabry; Alfa-galactosidasa; Enfermedad renal crónica. (fuente: DeCS BIREME)

\begin{abstract}
Fabry disease is a lysosomal storage disease caused by alpha galactosidase A enzyme deficiency. The pattern of inheritance is X-linked. The clinical presentation has a variety of symptoms and signs; and a classic clinical variant and non-classical clinical variants such as renal or cardiac have been described. The diagnosis is clinical and laboratory and even molecular and in some cases histology of the compromised organ is needed. The treatment in recent years remains symptomatic therapy and with enzyme replacement therapy; although chaperones are already available for the treatment of a group of patients with "amenable" mutations and they are studying gene therapy and nanotechnology. It must have a multidisciplinary approach, given the variety of symptoms and signs in its presentation. Early detection and early initiation of therapy could improve quality of life and limit the progression of the disease; and finally genetic counseling should always be considered.
\end{abstract}

Key words: Fabry disease, Alpha galactosidase, Chronic kidney disease. (source: MeSH NLM)

\section{INTRODUCCIÓN}

La enfermedad de Fabry (EF), llamada también enfermedad de Fabry-Anderson, se clasifica en la base de datos digital de la Herencia Mendeliana en el Hombre (OMIM, Online Mendelian Inheritance in Man)1 con el código \#301500; es una enfermedad hereditaria de depósito lisosomal causada por mutaciones específicas en el gen GLA, localizado en el cromosoma $X$, que producen deficiencia o ausencia de la enzima alfa-galactosidasa $\mathrm{A} 2$.
La EF, se caracteriza por el depósito de glicolípidos, principalmente globotriaosilceramida (Gb3 o GL3) y globotriaosilesfingosina (liso-Gb3 o liso-GL-3), en diferentes tejidos y órganos, como endotelio vascular, nervios periféricos, piel, aparato digestivo, córnea y en el sistema nervioso central, corazón y riñones ${ }^{3}$.

\section{HISTORIA}

En el año 1898 dos médicos dermatólogos -Johan Fabry, en Alemania, y William Anderson, en Gran

'Departamento de Nefrología, Hospital Nacional Edgardo Rebagliati Martins. ESSALUD, Lima-Perú.

Citar como: Martín Gómez-Luján. Enfermedad de Fabry. [Artículo de Revisión].2018;18(3):75-83. (Julio 2018). DOI 10.25176/RFMH.v18.n3.1595

Journal home page: http://revistas.urp.edu.pe/index.php/RFMH

(c) Los autores. Este artículo es publicado por la Revista de la Facultad de Medicina Humana, Universidad Ricardo Palma. Este es un artículo de Open Access distribuido bajo los términos de la Licencia Creative Commons Atribución-NoComercial-Compartirlgual 4.0 Internacional.(http://creativecommons.org/licenses/by-nc-sa/4.0/), que permite el uso no comercial, distribución y reproducción en cualquier medio, siempre que la obra original sea debidamente citadas. Para uso comercial, por favor póngase en contacto con revista.medicina@urp.pe 
Bretaña- reportaron, independientemente, casos de pacientes con lesiones dermatológicas denominadas como angioqueratomas corporales difusos ${ }^{4,5}$.

En 1947, se estableció que la EF se trataba de una enfermedad por acúmulo o depósito, ya que en todos los órganos afectados se encontraban vacuolas intracelulares de aspecto espumoso ${ }^{6}$.

Posteriormente, en 1963 Sweeley y cols. identificaron que el material acumulado en esas vacuolas fue un glucoesfingolípido7.

En 1967, Brady y cols. identificaron que la causa de la enfermedad era el déficit de la enzima responsable del catabolismo de la GL-3, la a-galactosidasa (a-GAL). Este descubrimiento permitió realizar el diagnóstico de la enfermedad mediante la determinación de los niveles plasmáticos de la a-GAL ${ }^{8}$.

En 1989, se identificó y secuenció el gen que codifica la a-GAL; este gen se localiza en el brazo largo del cromosoma X (Xq22.1). Este descubrimiento permitió el estudio molecular de la enfermedad, lo que culminó en el desarrollo de la terapia de reemplazo con enzima recombinante ${ }^{2,6}$.

\section{PREVALENCIA}

La EF es la segunda enfermedad más frecuente por depósito lisosomal después de la enfermedad de Gaucher².

En general se reporta incidencias entre 1 en 476000 a 1 en 117.000 en la población en general ${ }^{9,10}$.

Las mutaciones asociadas con las manifestaciones clásicas de la enfermedad de Fabry están presentes en aproximadamente 1:22,000 a 1:40,000 en hombres ${ }^{11,12}$, y las mutaciones asociadas con las presentaciones atípicas, llamadas de "inicio tardío", están presentes en aproximadamente 1:1000 a 1:3000 hombres y entre 1: 6000 a 1:40,000 en mujeres ${ }^{11-16}$.

La prevalencia de la enfermedad de Fabry esta subestimada por diferentes razones como: las manifestaciones de la enfermedad son variadas, dado que es una enfermedad rara o huérfana, el diagnóstico a menudo no es considerado y hay una demora en establecer el diagnóstico definitivo ${ }^{9}$. Así, Mehta y Cols ${ }^{17}$ realizaron una encuesta de EF entre 366 pacientes de once países de Europa y encontraron una demora media para corregir el diagnóstico después del inicio de los síntomas, la cual se estimó en 13,7 y 16,3 años para hombres y mujeres, respectivamente.

\section{HERENCIA}

Su transmisión está ligada al cromosoma X. El gen GLA que codifica para la enzima alfa galactosidasa se localiza en la banda Xq22.1 del brazo largo de dicho cromosoma $^{6,9,18}$. Tiene 12,000 pares de bases (pb) y contiene siete exones con un tamaño inferior a 300 pb cada uno ${ }^{6}$. Se han identificado más de 599 mutaciones en el gen de la a-GAL, que se distribuyen en los siete exones ${ }^{19}$. La mayoría de las mutaciones están restringidas a familias específicas, sin existir una clara relación genotipo-fenotipo ${ }^{9,19}$.

Por la forma en que se hereda la enfermedad, los hombres, que tienen un único cromosoma $X$, no pueden transmitir la condición a sus hijos. Sin embargo, todas las hijas tendrán una copia del gen defectuoso. Las mujeres tienen dos cromosomas $\mathrm{X}$, uno de los cuales tendrá una copia del gen defectuoso. Las mujeres tienen la probabilidad del $50 \%$ de poder transmitir el gen defectuoso a su descendencia, independientemente de que si el hijo es niño o niña ${ }^{3,9}$ (Figura 1).

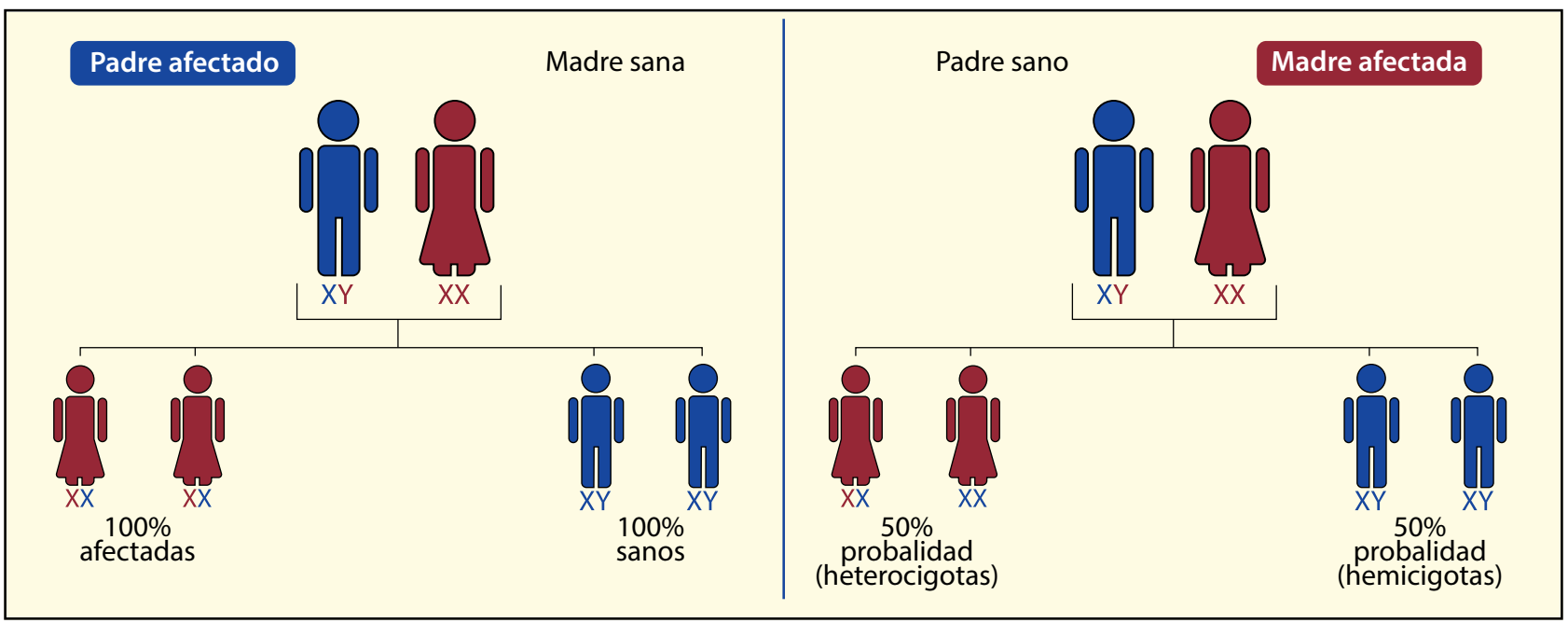

Figura 1. Patrón hereditario de la Enfermedad de Fabry. Modificado de García Trabanino R.3. 
En los varones (hemicigotos) se observa una alta penetrancia, aunque la expresión fenotípica del defecto enzimático presenta amplias variaciones intra e interfamiliares6,9. Las mujeres, consideradas antiguamente solo como portadoras, también pueden verse afectadas y en algunas ocasiones pueden presentar formas floridas de la enfermedad como consecuencia de la inactivación no aleatoria del cromosoma X sano; que corresponde al efecto Lyon ${ }^{20}$.

\section{FISIOPATOLOGÍA}

Los glicoesfingolípidos son componentes de las membranas plasmáticas, principalmente de los eritrocitos, hepatocitos y células epiteliales renales. Estas macromoléculas son sintetizadas a nivel hepático, luego se incorporan en las partículas de lipoproteínas para ser trasportadas en la circulación sistémica hacia las células donde serán integradas en las membranas. El 25\% de estos glicoesfingolípidos plasmáticos son sintetizados cada día y el resto deriva del recambio de los eritrocitos senescentes ${ }^{6,9}$. Finalmente los glicoesfingolípidos son endocitados en las células y degradados en los lisosomas 6 ,17,18 (Figura 2).

El catabolismo de estas complejas macromoléculas requiere de la acción de varias enzimas hidrolíticas, principalmente la a-GAL. Esta enzima hidrolítica se sintetiza como un precursor proteico de 429 aminoácidos ${ }^{17}$. Un porcentaje de la enzima fosforilada es secretada de las células y recaptada por endocitosis. Esta última acción es mediada por receptores transmembrana específicos (receptores manosa-6fosfato) $)^{9,19}$.

Al existir una deficiencia cuantitativa de la a-GAL, los glicoesfingolípidos -especialmente la Gb3- no se metabolizan y se acumulan dentro de los lisosomas en diversas estirpes celulares, incluyendo células endoteliales, pericitos, epitelio renal, miocardiocitos, neuronas y células de la córnea. La acumulación lisosomal de esta macromolécula da como resultado disfunción tanto lisosomal como celular, desencadenando una respuesta tisular inflamatoria que, finalmente, se traduce en isquemia tisular y celular crónica, y daño multisistémico irreversible ligado al depósito lisosomal ${ }^{9,17}$. (Figura 2).

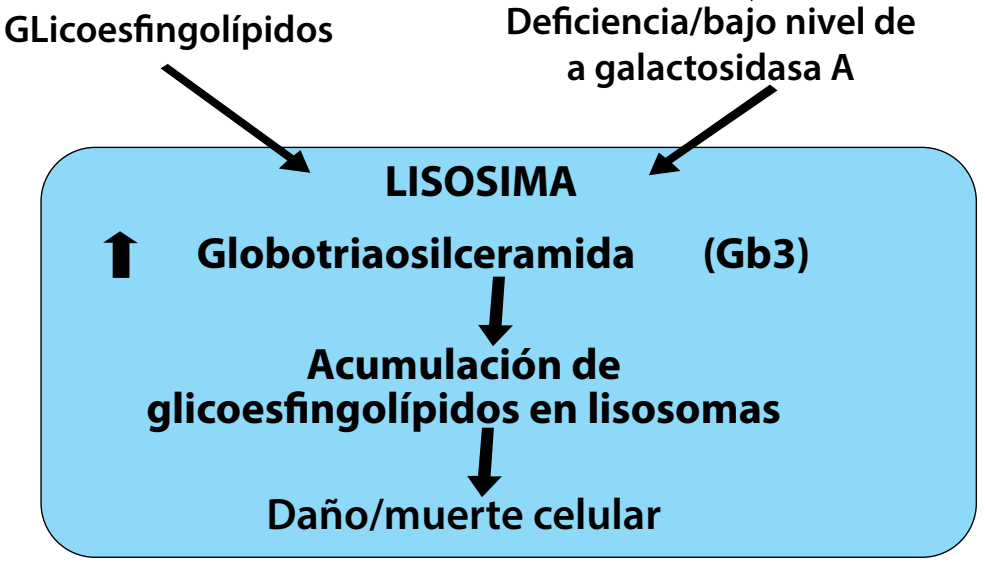

Figura 2. La deficiencia de la alfa galactosidasa A resulta en defectos en la degradación de los glicoesfingolípidos y lleva a una anormal acumulación de Globotriaosilceramida. Modificado de El-Abassi R.18.

La acumulación de Gb3 en las células del endotelio vascular da lugar a la disminución progresiva de la luz vascular hasta ocluirla por completo, alterando la reactividad vascular y generando un estado protrombótico21. Todo en conjunto origina eventos isquémicos que afectan varios sitos del organismo (riñón, corazón, cerebro, intestinos, etc.), los eventos cardiovasculares (principalmente la cardiopatía isquémica y los accidentes cerebrovasculares) son las causas de mortalidad más frecuentes de estos pacientes22,23. Otro mecanismo que contribuye al daño celular en esta enfermedad es la composición alterada de los lípidos de la membrana celular, lo cual da lugar a alteraciones en el transporte y almacenamiento de las proteínas transmembrana asociadas a las balsas lipídicas, traduciéndose en anormalidades en el transporte de micro y macromoléculas ${ }^{9,24}$. 


\section{MANIFESTACIONES CLÍNICAS}

Se han descrito dos formas clínicas o fenotipos de la enfermedad: la forma clásica o severa y la llamada variante no clásica o de inicio tardío, donde el daño se manifiesta principalmente en tejido renal o cardíaco, con complicaciones cerebrovasculares secundarias ${ }^{2}$ 9,22-25 (Tabla 1).

Tabla 1. Fenotipos de la Enfermedad de Fabry.

\section{EXPRESIÓN CLÍNICA}

FENOTIPO CLÁSICO

FENOTIPO NO CLÁSICO
Acroparestesias

\section{Córnea verticilata}

Angioqueratomas

Hipoanhidrosis

Molestias gastrointestinales

Microalbuminuria/Proteinuria

Hipertrofia Ventricular Izquierda

Accidente cerebrovascular

Actividad enzimática residual
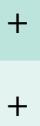

$+$
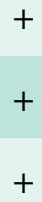

$+$

$+$

$<5 \%$
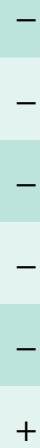

$+$

$+($ a)

$\geq 5 \%$

(a) No se ha reportado una variante de inicio del adulto primariamente cerebrovascular, es secundario a cardiopatía.

Fuente: Modificado de García-Trabanino $\mathbf{R}^{3}$.

La enfermedad de Fabry es un trastorno clínicamente heterogéneo que produce una amplia variedad de manifestaciones, por lo que el cuadro clínico puede ser muy diverso $0^{3,26,27}$.

En la variante clásica es común encontrar desde la infancia dolores neuropáticos, angioqueratomas y córnea verticilada. Con el tiempo, los pacientes desarrollan enfermedad renal crónica y enfermedad cardíaca o de otros órganos, llegando habitualmente a necesitar terapia de reemplazo renal generalmente en la cuarta década de la vida; sin embargo, cuando se realiza el diagnóstico temprano de puede evitar mayores daños e incluso ser reversible, sino se incrementa la mortalidad $28,29,30,31$. En la variante no clásica los pacientes pueden transcurrir completamente asintomáticos hasta la edad adulta, cuando se manifiestan las complicaciones graves, habitualmente renales y cardíacas ${ }^{26,27,32}$.

\section{Manifestaciones neurológicas}

El dolor neuropático, denominado acroparestesias, suele ser el síntoma inicial desde muy temprana edad. El paciente suele referirlo como ardor, quemazón, electricidad $u$ hormigueo en manos $y / o$ pies, de intensidad variable desde leve-moderado hasta intenso y crisis de dolor que puede incapacitarlo y afectar severamente su calidad de vida, su asistencia a su centro educativo, a su centro laboral y su aspecto social ${ }^{9,28}$. Estas crisis se desencadenan típicamente durante el ejercicio, estados febriles y generalmente con los cambios en la temperatura del ambiente. El examen neurológico suele ser normal al inicio. El electromiograma y las velocidades de conducción motoras y sensitivas son normales ${ }^{29}$. Las manifestaciones gastrointestinales muchas veces son inespecíficas, pero son frecuente y de intensidad variable e incluso se han presentado con cuadros intensos de dolor abdominal podrían confundirse con cuadros quirúrgicos; se ha postulado el mecanismo del acúmulo de Gb3 y liso-Gb3 en los ganglios submucosos y mientéricos; y por eso los pacientes generalmente presentan diarrea, dolor abdominal tipo cólico náuseas, saciedad temprana, vómitos y/o estreñimiento, ${ }^{933-35}$.

Luego de la tercera década de vida pueden persistir el dolor neuropático y la disautonomía, manifestada como déficit en la vasorreactividad cerebral, síncopes y ortostatismo, además del compromiso del sistema nervioso central, manifestado en eventos cerebrovasculares isquémicos (transitorios o infartos) y/o hemorrágicos, habitualmente a partir de la cuarta década de la vida ${ }^{36}$. Este mecanismo de daño puede ser silente y diagnosticarse como hallazgo incidental 
en una resonancia magnética cerebral, con o sin dolicoectasia vertebro-basilar ${ }^{36,37}$.

\section{Manifestaciones cardiológicas}

La cardiopatía se manifiesta hipertrofia ventricular izquierda (HVI) y arritmias, y generalmente progresa a insuficiencia cardíaca ${ }^{37}$. La HVI no es consecuencia del acúmulo de Gb3, sino del estímulo hipertrófico de este sustrato. De hecho, en los pacientes afectados el Gb3 representa solo el $1-2 \%$ de la masa cardíaca ${ }^{38}$. Las manifestaciones tempranas suelen aparecer antes de los 35 años en forma de trastornos de la conducción como bradicardia, bloqueos de rama o acortamiento del intervalo $\mathrm{PR}^{9,39}$.

Luego de la tercera década de vida se pueden presentar cuadros anginosos asociados a disnea, palpitaciones y mareos. La disautonomía cardíaca es común en esta etapa. La HVI condiciona disfunción diastólica, progresando luego a fallo sistólico y fibrosis endomiocárdica ${ }^{9,38}$. Los trastornos de conducción y alteraciones de la repolarización ventricular izquierda se vuelven más evidentes y es posible la aparición de arritmias malignas que necesiten marcapasos ${ }^{39,40}$.

La detección de casos a través de cardiología debe implementarse dado que hay casos de pacientes con cardiopatías no filiadas y que podrían corresponder a la variante cardíaca de la enfermedad de Fabry ${ }^{41}$.

\section{Manifestaciones renales}

La nefropatía en la enfermedad de Fabry puede manifestarse inicialmente como isostenuria, signos de disfunción tubular e hiperfiltración con o sin albuminuria. Hay estudios en que la podocituria es un biomarcador de daño renal más temprano; sin embargo, su uso no es habitual en la práctica clínica ${ }^{41,42}$. La EF progresa lenta y progresivamente a ERC y cuando alcanza el estadio 5; puede requerir terapia de reemplazo renal (TRR) y en algunos casos trasplante renal ${ }^{43}$. La progresión es más frecuente en varones, aunque algunas mujeres también llegan a requerir $\mathrm{TRR}^{9,32}$. La mitad de los pacientes varones con fenotipo clásico presentarán compromiso renal antes de los 35 años y todos después de los 50 años ${ }^{44}$.

Se ha descrito la presencia de albuminuria desde muy temprana edad, incluso desde los 6 años, en algunos hemicigotos con fenotipo clásico ${ }^{45}$. La albuminuria es de rango variable y es la expresión más frecuente de lesión renal. Sin embargo, puede haber lesión renal sin proteinuria en el $10 \%$ de los varones y el $30 \%$ de las mujeres con ERC $3^{41}$. La albuminuria puede llegar a rango nefrótico en el $20 \%$ de los pacientes ${ }^{32}$.
Menos del 20\% de los pacientes presentan hematuria microscópica ${ }^{32}$.

La biopsia renal es importante, dado que conoce determinar el daño estructural renal, al realizarla es necesario utilizar tinción de hematoxilina-eosina y azul de toluidina en el estudio de microscopia de luz y se recomienda guardar una muestra de tejido en glutaraldehído para practicar microscopía electrónica, si está disponible, para evidenciar compromiso en los podocitos y puede tener impacto en el pronóstico y en la decisión terapéutica ${ }^{9,46-48}$.

En la enfermedad de Fabry, se producen depósitos renales de Gb3 en los podocitos, mesangio, endotelio del capilar glomerular, epitelio tubular, células endoteliales y de la capa muscular de arterias y arteriolas, y en las células intersticiales ${ }^{49}$

Muestra cambios característicos de la enfermedad: microscopia óptica (células espumosas), microscopía electrónica (cuerpos lamelares o con aspecto de cebra), depósito de GB3 característico con tinciones especiales $^{2,49}$.

\section{Manifestaciones oftalmológicas}

Como patología oftalmológica se han descrito: alteraciones vasculares, edema periorbitario, córnea verticilata, cataratas, pigmentación retiniana periférica, papiledema, oclusión de la arteria central de la retina, atrofia óptica, discromatopsia, nistagmus y oftalmoplejía internuclear ${ }^{50}$.

La afección corneal es la más frecuente. Lo más característico es el depósito de glucoesfingolípidos entre la membrana basal del epitelio corneal y la membrana de Bowman produciendo el patrón de afectación corneal en «córnea verticilada», que también se observa en pacientes afectos de enfermedad de Tangier, Melanoqueratosis estriada, Síndrome de Melkersson-Rosenthal y secundarios a fármacos como Amiodarona o Cloroquina. Los fenómenos oclusivos retinianos y la ceguera no son frecuentes y se ha correlacionado las manifestaciones clínicas con la severidad de la enfermedad ${ }^{50-52}$.

\section{Manifestaciones dermatológicas}

Dentro de las lesiones dermatológicas tenemos: los angioqueratomas, que aparecen desde la infancia o adolescencia. Por lo general se encuentran agrupados en la región glútea, la región umbilical, muslos y genitales. Los angioqueratomas diseminados son altamente indicativos de enfermedad de Fabry pero no patognomónicos. Puede realizarse biopsia de piel para descartar diagnósticos diferenciales de las lesiones; el 
estudio anatomopatológico dela biopsia de piel muestra dolicoectasia de vasos de la dermis con microtrombosis en su interior. Otras lesiones son las telangiectasias en piel y mucosas, se observan predominantemente en rostro, labios y mucosa oral ${ }^{9,53,54}$.

Se pueden presentar alteraciones en la sudoración: como la hipohidrosis o anhidrosis es frecuente y suele ser un signo precoz en la infancia, provocando piel seca, intolerancia al calor o al ejercicio y fiebre de origen desconocido.También puede disminuirsela producción de lágrimas y saliva ${ }^{3,9}$. Otras manifestaciones menos frecuentes son el linfedema, la disminución del vello corporal y la alopecia difusa ${ }^{55}$.

\section{Manifestaciones otorrinolaringológicas}

Entre las principales manifestaciones tenemos a la hipoacusia tipo perceptiva o neurosensorial, unilateral o bilateral, progresiva o de instalación súbita (en el lapso de 72 h), de grado leve a severa9,56. El perfil audiométrico no es patognomónico y puede comprometer las frecuencias agudas o ser pantonal. Los acúfenos pueden ser unilaterales o bilaterales, esporádicos o permanentes, de tonalidad aguda. El vértigo se presenta en crisis espontáneas de breve duración y puede está asociado a la pérdida auditiva; en algunos casos se presenta con sordera súbita en frecuencias agudas ${ }^{9,56}$.

Estas manifestaciones son el resultado del compromiso del oído interno, tanto del laberinto anterior (audición) como del posterior (equilibrio), y de la vía neurológica auditiva (octavo par craneal, tronco cerebral y corteza auditiva $)^{9,56}$.

\section{Manifestaciones cognitivas y psicológicas}

Dentro de las manifestaciones cognitivas y psicológicas podemos encontrar a alteraciones en las áreas del razonamiento, resolución de problemasy perseveración y son más propensos a presentar síntomas de ansiedad y depresión y dificultades a la adaptación social; el análisis de las pruebas correlacionales indicaron un vínculo entre las medidas cognitivas y clínicas de la gravedad de la enfermedad ${ }^{57}$.

Las manifestaciones clínicas requieren de un control y seguimiento de los síntomas y signos, la frecuencia del seguimiento va a depender del órgano comprometido y debería ser por un equipo multidisciplinario.

\section{DIAGNÓSTICO}

El diagnóstico definitivo de la enfermedad de Fabry en varones con fenotipo clásico se establece al determinar deficiencia o ausencia de la actividad de la alfa-galactosidasa $A$ en leucocitos aislados de sangre periférica ${ }^{9,58}$

La determinación enzimática puede también realizarse en gotas de sangre seca en papel filtro (dried blood spots, DBS). Esto permite el envío de muestras a distancia, el diagnóstico retrospectivo y el tamizaje poblacional ${ }^{59}$. No obstante, un resultado anormal en la prueba del papel filtro, DBS, debe confirmarse con la prueba en leucocitos o mediante estudio molecular ${ }^{9,58}$.

En las mujeres, debido a la inactivación aleatoria de uno de los cromosomas $X$, la actividad enzimática de la alfa-galactosidasa A puede ser normal hasta en el $40 \%$ de los casos (falso negativo). Por tanto, un valor normal de actividad enzimática no descarta la enfermedad en mujeres y para el diagnóstico debe hacerse estudio genético ${ }^{9,60}$.

Es importante considerar que la medición de la actividad enzimática también puede producir falsos positivos, es decir, puede haber actividad enzimática disminuida en personas sanas portadoras de variantes genéticas de significado incierto o de polimorfismos no patogénicos ${ }^{9,61}$. En estos pacientes la sola demostración de valores de actividad enzimática disminuida no es indicación de inicio de terapia de reemplazo enzimático sin haber realizado antes una confirmación molecular o, en casos excepcionales, una determinación de biomarcadores como Gb3 y LisoGb3 en plasma u orina o en tejidos mediante biopsia, u demostrar lesiones histológicas en relación al cuadro clínico ${ }^{62,63}$.

En el diagnóstico molecular debe identificarse cualquiera de las múltiples mutaciones responsables de la enfermedad. En varones con fenotipo clásico, el estudio de la secuencia codificante del gen GLA para identificar la mutación patogénica es una prueba diagnóstica complementaria ${ }^{9,62}$.

La identificación de la mutación es particularmente útil para el estudio familiar y constituye la prueba confirmatoria definitiva en mujeres sintomáticas con actividad enzimática normal ${ }^{9,58}$.

\section{TRATAMIENTO}

Se dispone de terapia de reemplazo enzimática y el uso de chaperonas químicas para el tratamiento para la enfermedad de Fabry. Otros tratamientos están siendo desarrollados y evaluados como la terapia génica, inhibición del sustrato y el uso nanotecnología. Se considera que todo varón que presente la variante clásica debe recibir tratamiento independiente de la edad $^{64}$. Así mismo, deberían considerarse para la terapia 
si los hallazgos de laboratorio e imágenes evidencian daño de órgano como el ojo, riñón, el corazón o el cerebro y sistema nervioso; con o sin deterioro de la calidad de vida; así como a los pacientes que se encuentran en lista de espera para un trasplante ${ }^{64}$.Sin embargo, debe individualizarse cada paciente para decidir la terapéutica.

El uso de chaperonas químicas, Migalastat, una nueva clase de pequeñas moléculas que actúan mediante la estabilización de proteínas inestables, ya está aprobado en Europa como clorhidrato de migalastat, para su uso vía oral y en un grupo especial de pacientes que tienen mutaciones "susceptibles" en el gen de la alfa-galactosidasa $A^{65-67}$.

Las chaperonas farmacológicas son pequeñas moléculas que ayudan a mejorar el plegamiento de las moléculas proteicas en general. Las chaperonas actúan sobre una proteína, en este caso la enzima anómala que presenta una conformación que no es la que debería tener, pegándose a ella, acompañándola y cambiándole la conformación. Así el fármaco se acopla a ciertas formas inestables de la alfa-galactosidasa A, estabilizando la enzima. Esto permite que la enzima se transporte a zonas de la célula en la que puede descomponer la GL-365.

Por tanto, para poder ofrecer este tratamiento necesitamos que haya proteína, es decir, necesitamos que los pacientes tengan al menos codificada una proteína y eso va en relación con el tipo de mutaciones que debe tener el gen anómalo y que le permita actuar; se estima que un $30 \%$ de pacientes tendrían mutaciones "susceptibles" y en quienes se beneficiarían de este fármaco ${ }^{65,66}$.

Para la terapia de reemplazo enzimático se cuenta con la agalsidasa alfa y la agalsidasa beta. Ambas están compuestas por la misma cadena de aminoácidos, es decir, son la misma proteína humana. La diferencia reside en la dosis aprobada (agalsidasa beta $1 \mathrm{mg} / \mathrm{kg}$ de peso y agalsidasa alfa $0,2 \mathrm{mg} / \mathrm{kg}$ ) y en la cantidad de residuos de manosa 6-fosfato (agalsidasa beta $3,6 \mathrm{~mol} / \mathrm{mol}$ de proteína y agalsidasa alfa $1,3 \mathrm{~mol} / \mathrm{mol}$ de proteína) $)^{3,9,68}$.

Su principal mecanismo de acción es la sustitución de la enzima alfa-galactosidasa $A$, posibilitando la hidrólisis del Gb3 y liso-Gb3 al separar un residuo de galactosa terminal de la molécula, disminuyendo así su acumulación en los tejidos ${ }^{2,9}$.
La presencia de mayor cantidad de residuos de manosa ${ }^{6}$-fosfato produce un mejor reconocimiento por los receptores de manosa ${ }^{6}$-fosfato de la membrana celular y consecuentemente mayor internalización celular de la enzima9,66. De acuerdo con los estudios publicados, pareciera que el beneficio tisular y clínico tiene relación directa con la dosis de TRE utilizada9,67-72. Distintos protocolos han demostrado que el uso de agalsidasa beta a dosis de $1 \mathrm{mg} / \mathrm{kg}$ cada 14 días produce remoción total de Gb3 de los podocitos renales en comparación con la agalsidasa alfa a dosis de 0,2mg/kg9,70-72. Más aún, la reducción de la dosis de $1 \mathrm{mg} / \mathrm{kg}$ a 0,2mg/kg cada 14 días conduce a reacúmulo del sustrato en los vasos y podocitos luego de varios años, reapareciendo también los síntomas de la enfermedad que habían sido resueltos con el uso de dosis mayores ${ }^{71,74}$.

\section{CONCLUSIÓN}

Se debe tener un abordaje multidisciplinario para las evaluaciones clínicas, dado la variedad de síntomas y signos en su presentación.

La detección temprana y el inicio temprano de la terapia podrían mejorar o limitar la progresión de la enfermedad.

La terapéutica en pacientes con EF debería ser individualizada según las variantes clínicas y la severidad de los síntomas del paciente.

Debe considerarse la consejería genética en las familias de los casos detectados.

Contribuciones de autoría: El autor participó la generación, redacción y aprobación final del artículo original.

Financiamiento: Autofinanciado.

Conflicto de interés: El autor declara no tener conflicto de interés en la publicación de este articulo.

Recibido: 23 de marzo del 2018

Aprobado: 03 de mayo del 2018

Correspondencia: Martín Gómez Luján.

Dirección: Jr. Edgardo Rebagliati 490, Jesus María, Departamento de Nefrología, Hospital Rebagliati, EsSALUD, Jesús María, Lima - Perú. Celular: +511-975101783

Correo: martingomezlujan@hotmail.com 


\section{REFERENCIAS BIBLIOGRÁFICAS}

1. OMIM. Online Mendelian Inheritance in Man. Baltimore, Johns Hopkins University (actualizado al 18 Mayo 2018). Disponible en: www. omim.org

2. Germain D. Fabry disease. Orphanet J Rare Dis 2010;5:30.

3. García-Trabanino R, Badilla-Porras R, Carazo K, Courville K, De Luna E. et al. Consenso del Grupo Centroamericano y del Caribe para el Estudio y Tratamiento de la Enfermedad de Fabry. Nefrol Latinoam 2017;14(1): 27-38.

4. Fabry J. Ein Beitrag Zur Kenntnis der Purpura haemorrhagica nodularis. Arch Dermatol Syphilis 1898; 43:187-200.

5. Anderson W. A case of angioqueratoma. Br J Dermatol 1898; 10:113-7.

6. Vega-Vega O, Pérez-Gutierrez A, Correa-Rotter R. Enfermedad de Fabry-Anderson. Rev Invest Clin 2011; 63 (3): 314-321.

7. Sweeley C, Klionsky B. Fabry's Disease: Classification as a Sphingolipidosis and Partial Characterization of a Novel Glycolipid. J Biol Chem 1963; 238: 3148-50.

8. Brady R, Gal A, Bradley R, Martenesson E, Warshaw A, Laster L. Enzymatic defect in Fabry's disease: Ceramide trihexosidase deficiency N Engl J Med 1967; 276: 1163-7.

9. Consenso de Médicos de AADELFA y GADYTEFNeumann Antongiovanni N, Fainboim A, Kisinovsky I, Amartino H, Cabrera G, et al. Guidelines for diagnosis, monitoring and treatment of Fabry disease. Medicina (B Aires) 2013;73(5):482-94.

10. Meikle P, Hopwood J, Clague A, Carrey W. Prevalence of lisosomal storage disorders. JAMA 1999; 281:249-254.

11. Spada M, Pagliardini S, Yasuda M, Tukel T, Thiagarajan G, Sakuraba H, et al. High incidence of later-onset fabry disease revealed by newborn screening. Am J Hum Genet 2006;79(1):31-40.

12. Hwu W, Chien $Y$, Lee N, Chiang S, Dobrovolny R, Huang A, Yeh $\mathrm{H}_{\text {, }}$ Chao M, Lin S, Kitagawa T, Desnick R, Hsu L. Newborn screening for Fabry disease in Taiwan reveals a high incidence of the later-onset GLA mutation c.936+919G>A (IVS4+919G>A). Hum Mutat 2009;30(10):1397-405.

13. Mechtler T, Stary S, Metz T, De Jesús V, Greber-Platzer S, Pollak A Herkner K, Streubel B, Kasper D. Neonatal screening for lysosomal storage disorders: feasibility and incidence from a nationwide study in Austria. Lancet 2012; 28;379(9813):335-41

14. Lin H, Chong K, Hsu J, Yu H, Shih C, Huang C, Lin S, Chen C, Chiang C, Ho H, Lee P, Kao C, Cheng K, Hsueh C, Niu D. High Incidence of the Cardiac Variant of Fabry Disease Revealed by Newborn Screening in the Taiwan Chinese Population. Circ Cardiovasc Genet 2009;2(5):450-6.

15. Inoue T, Hattori K, Ihara K, Ishii A, Nakamura K, Hirose S. Newborn screening for Fabry disease in Japan: prevalence and genotypes of Fabry disease in a pilot study. J Hum Genet 2013;58(8):548-52.

16. Chien Y, Lee N, Chiang S, Desnick R, Hwu W. Fabry Disease: Incidence of the Common Later-Onset a Galactosidase A IVS4+919G囚A Mutation in Taiwanese Newborns-Superiority of DNA-Based to Enzyme-Base Newborn Screening for Common Mutations. Mol Med 2012;18:780-784

17. Mehta A, Ricci R, Widmer U, Dehout F, Garcia de Lorenzo A, Kampmann C, Linhart A, Sunder-Plassmann G, Ries M, Beck M. Fabry disease defined: baseline clinical manifestations of 366 patients in the Fabry Outcome Survey. Eur J Clin Invest. 2004 Mar;34(3):236-42.

18. El-Abbassi R, Singjal D, England J. Fabry Disease. Journal of the Neurological Sciences 2014; 344: 5-19.

19. Olivera-González S, Josa-Laorden C, Torralba-Cabeza MA. Fisiopatología de la enfermedad de Fabry. Rev Clin Esp. 2018;218(1):22-28.

20. Lyon M. Some milestones in the history in the history of X-chromosome inactivation. Annu Rev Genet 1992;26:17-28.

21. De Graba T, Azhar S, Dignat-George F, Brown E, Boutiére B, Altarescu
G, McCarron R, Schiffmann R. Profile of Endothelial and Leukocyte Activation in Fabry Patients. Ann Neurol 2000;47:229-233.

22. Kampmann C, Baehner F, Ries M, Beck M. Cardiac involvement in Anderson-Fabry disease. J Am Soc Nephrol 2002;13(Supl. 2): S147-S149.

23. Kolodny EH, Pastores GM. Anderson-Fabry disease: extrarenal, neurologic manifestations. J Am Soc Nephrol 2002;13(Supl. 2): S150-S153.

24. Pastores GM, Lien YH. Biochemical and molecular genetic basis of Fabry disease. J Am Soc Nephrol 2002; 13(Suppl. 2):130-3.

25. Ferri $L$ et al. Fabry disease: polymorphic haplotypes and a novel missense mutation in the GLA gene. Clinical Genetic 2012;82:224-233.

26. Marchesoni CL, Roa N, Pardal AM, Neumann P, Cáceres G, Martínez P, et al. Misdiagnosis in Fabry disease. J Pediatr 2010;156(5):828-31.

27. Serebrinsky G, Calvo M, Fernandez S, Saito S, Ohno K, Wallace E, et al. Late onset variants in Fabry disease: Results in high risk population screenings in Argentina. Mol Genet Metab Rep 2015;4:19-24.

28. Politei JM, Pagano MA. Peripheral neuropathy in AndersonFabry disease: Its physiology, evaluation and treatment. Rev Neurol 2004;38(10):979-83.

29. Burlina AP, Sims KB, Politei JM, Bennett GJ, Baron R, Sommer C, et al. Early diagnosis of peripheral nervous system involvement in Fabry disease and treatment of neuropathic pain: The report of an expert panel. BMC Neurol 2011;11:61.

30. Waldek S, Patel MR, Banikazemi M, Lemay R, Lee P. Life expectancy and cause of death in males and females with Fabry disease: Findings from the Fabry Registry. Genet Med 2009;11(11):790-6.

31. Ortiz A, Oliveira J, Waldek S, Warnock D, Cianciaruso B, Wanner C. Fabry Registry. Nephropathy in males and females with Fabry disease: Cross-sectional description of patients before treatment with enzyme replacement therapy. Nephrol Dial Transplant 2008;23(5):1600-7.

32. Ortiz A, Oliveira J, Waldek S, Warnock D, Cianciaruso B, Wanner C. Fabry Registry. Nephropathy in males and females with Fabry disease: Cross-sectional description of patients before treatment with enzyme replacement therapy. Nephrol Dial Transplant 2008;23(5):1600-7.

33. Politei J, Thurberg BL, Wallace E, Warnock D, Serebrinsky G, Durand $C$, et al. Gastrointestinal involvement in Fabry disease. So important, yet often neglected. Clin Genet 2016;89(1):5-9.

34. Wilcox W, Feldt-Rasmussen U, Martins A, Ortiz A, Lemay R, Jovanovic A, Germain D, Carmen Varas, Nicholls K, Weidemann F, HopkinGg R. Improvement of Fabry Disease-Related Gastrointestinal Symptoms in a Significant Proportion of Female Patients Treated with Agalsidase Beta: Data from the Fabry Registry.JIMD Rep 2018;38:45-51.

35. Hilza M, Arbustini E, Dagna L, Gasbarrini A, Goizetf C, Lacombe D, Liguorih R, Manna R, Politei J, Spada M, Burlina A. Non-specific gastrointestinal features: Could it be Fabry disease?. Digestive and Liver Disease 2018;50(5):429-437.

36. Kolodny E, Fellgiebel A, Hilz MJ, Sims K, Caruso P, Phan TG, et al. Cerebrovascular involvement in Fabry disease: Current status of knowledge. Stroke 2015;46(1):302-13.

37. Feldt-Rasmussen U. Fabry disease and early stroke. Stroke Res Treat 2011; 615218. doi:10.4061/2011/615218

38. Strotmann J, Breunig F, Wanner C, Weidemann F. Progression of Fabry cardiomyopathy. Clin Ther 2007;29 Suppl A. S13-4.

39. Tschöpe C, Dominguez F, Canaan-Kühl S, Blaschke D, Kühl U, Pieske $\mathrm{B}$, et al. Endomyocardial biopsy in Anderson-Fabry disease: The key in uncertain cases. Int J Cardiol 2015;190:284-6.

40. Namdar M. Electrocardiographic changes and arrhythmia in Fabry disease. Front Cardiovasc Med 2016;3:7. 
41. Savary A, Morello R,Brasse-Lagnel C, Milliez P, Bekri S, Labombarda F. Enhancing the diagnosis of fabry disease in cardiology with a targeted information: a before-after control-impact study. Open Heart 2017;4:e000567. doi:10.1136/openhrt-2016-000567.

42. Trimarchi H, Canzonieri R, Muryan A, Schiel A, Araoz A, Forrester $M$, et al. Copious podocyturia without proteinuria and with norma renal function in a young adult with Fabry disease. Case Rep Nephrol 2015;2015:257628.

43. Schiffmann R, Warnock DG, Banikazemi M, Bultas J, Linthorst GE, Packman S, et al. Fabry disease: Progression of nephropathy, and prevalence of cardiac and cerebrovascular events before enzyme replacement therapy. Nephrol Dial Transplant 2009;24(7):2102-11.

44. Wanner C, Oliveira JP, Ortiz A, Mauer M, Germain DP, Linthorst GE, et al. Prognostic indicators of renal disease progression in adults with Fabry disease: Natural history data from the Fabry Registry. Clin J Am Soc Nephrol 2010;5(12):2220-8.

45. Hopkin RJ, Bissler J, Banikazemi M, Clarke L, Eng CM, Germain DP et al. Characterization of Fabry disease in 352 pediatric patients in the Fabry Registry. Pediatr Res 2008;64(5):550-5.

46. Pereira EM, Silva AS, Labilloy A, Monte Neto JT, Monte SJ. Podocyturia in Fabry disease. J Bras Nefrol 2016;38(1):49-53.

47. Trimarchi. Expression of UPAR in Urinary Podocytes of Patients with Fabry Disease. International Journal of Nephrology 2017:1287289. https://doi.org/10.1155/2017/1287289

48. Trimarchi $H$, Canzonieri R, Schiel A, Costales-Collaguazo C, Politei C, Stern A, Paulero M, Rengel T, Andrews J, M. Forrester1, M. Lombi1, V. Pomeranz, R. Iriarte, Muryan A, Zotta E, Sanchez-Niño M, Ortiz A Increased urinary CD80 excretion and podocyturia in Fabry disease. Transl Med 2016;14:289.

49. Fogo A, Bostad L, Svarstad E, Cook WJ, Moll S, Barbey F, Geldenhuys L, West M, Ferluga D, Vujkovac B, Howie A, Burns A, Reeve R, Waldek $S$, Noël L, Grünfeld J, Valbuena C, Oliveira J, Müller J, Breunig F, Zhang $X$. Scoring system for renal pathology in Fabry disease: report of the International Study Group of Fabry Nephropathy (ISGFN). Nephrol Dia Transplant. 2010 Jul;25(7):2168-77.

50. Rodríguez-González-Herrero M, Marín-Sánchez J, Gimeno J, MoleroIzquierdo C, De-Casas-Fernández A, Rodríguez-González-Herrero B, SanRomán I, Lozano J, De-la-Morena G, Llovet-Osuna F. Manifestaciones oftalmológicas en la enfermedad de Fabry: a propósito de 4 casos con actividad deficiente de a-galactosidasa. Arch Soc Esp Oftalmol 2008; 83: 713-718.

51. Nguyen T, Gin T, Nicholls K, Low M, Galanos J, Crawford A.Ophthalmological manifestations of Fabry disease: a survey of patients at the Royal Melbourne Fabry Disease Treatment Centre. Clin Exp Ophthalmol 2005;33(2):164-8.

52. Pitz S, Kalkum G, Arash L, Karabul N, Sodi A, Larroque S, Beck M, Andreas Gal A. Ocular signs correlate well with disease severity and genotype in Fabry Disease. PLoS One 10(3): e0120814. doi:10.1371/ journal.pone.0120814.

53. Morais $P$, Santos AL, Baudrier $T$, Mota AV, Oliveira JP, Azevedo $F$. Angiokeratomas of Fabry successfully treated with intense pulsed light. J Cosmet Laser Ther 2008;10(4):218-22.

54. Amann-Vesti B, Gitzelmann G, Widmer U, Bosshard NU, Steinmann B Koppensteiner R. Severe lymphatic microangiopathy in Fabry disease. Lymphat Res Biol 2003;1(3):185-9.

55. Pistone G, Rizzo D, Bongiorno M. Cutaneous complications of Anderson-Fabry disease. Curr Pharm Des. 2013;19(33):6031-6.

56. Germain D, Avan P, Chassaing A, Bonfils P. Patients affected with Fabry disease have an increased incidence of progressive hearing loss and sudden deafness: An investigation of twenty-two hemizygous male patients. BMC Med Genet 2002;3:10.
57. Sigmundsdottir L, Tchan M, Knopman A, Menzies G, Batchelor J, Sillence O. Cognitive and Psychological Functioning in Fabry Disease. Archives of Clinical Neuropsychology 29 (2014) 642-650.

58. Gal A, Hughes DA, Winchester B. Toward a consensus in the laboratory diagnostics of Fabry disease -recommendations of a European expert group. J Inherit Metab Dis 2011;34(2):509-14.

59. Chamoles N, Blanco M, Gaggioli D. Fabry disease: enzymatic diagnosis in dried blood spots on filter paper. Clin Chim Acta 2001;308(1-2):195-6.

60. Linthorst GE, Poorthuis BJ, Hollak CE. Enzyme activity for determination of presence of Fabry disease in women results in $40 \%$ false-negative results. J Am Coll Cardiol 2008;51(21):2082.

61. Froissart R, Guffon N, Vanier MT, Desnick RJ, Maire I. Fabry disease: $\mathrm{D} 313 \mathrm{Y}$ is an alpha-galactosidase A sequence variant that causes pseudodeficient activity in plasma. Mol Genet Metab 2003;80(3):307-14. 62. Ouyang Y, Chen B, Pan X, Wang Z, Ren H, Xu Y, Ni L, Yu X, Yang L, Chen $\mathrm{N}$. Clinical significance of plasma globotriaosylsphingosine levels in Chinese patients with Fabry disease. Exp Ther Med 2018 15(4):3733-3742.

63. Niemann M, Rolfs A, Störk S, Bijnens B, Breunig F, Beer M, et al. Gene mutations versus clinically relevant phenotypes: Lyso-Gb3 defines Fabry disease. Circ Cardiovasc Genet 2014;7(1):8-16.

64. Ortiz A, Germain D, Desnickc R, Politei J, Mauer M, Burlinaf A et al, Fabry disease revisited: Management and treatment recommendations for adult patients. Molecular Genetics and Metabolism 2018;123(4):416-427.

65. Hughes D, Nicholls K, Shankar S, Sunder-Plassmann G, Koeller D, Nedd K, Vockley $G$ et al. Oral pharmacological chaperone migalastat compared with enzyme replacement therapy in Fabry disease: 18-month results from the randomised phase III ATTRACT study. J Med Genet. 2017 Apr; 54(4): 288-296.

66. Germain D, Hughes D, Nicholls K, Bichet D, Giugliani R, Wilcox W et al. Treatment of Fabry's Disease with the Pharmacologic Chaperone Migalastat. N Engl J Med 2016 ;375(6):545-55.

67. Lukas J, Giese A-K, Markoff A, Grittner U, Kolodny Ed, Mascher H et al. Functional characterisation of alpha-galactosidase $A$ mutations as a basis for a new classification system in Fabry disease. PLoS Genet 9(8): e1003632. doi:10.1371/journal.pgen.1003632.

68. Politei J, Schenone AB, Cabrera G, Heguilen R, Szlago M. Fabry disease and enzyme replacement therapy in classic patients with same mutation: Different formulations — different outcome? Clin Genet 2016;89(1):88-92.

69. Tondel C, Kanai T, Larsen KK, Ito S, Politei JM, Warnock DG, et al. Foot process effacement is an early marker of nephropathy in young classic Fabry patients without albuminuria. Nephron 2015;129(1):16-21.

70. Tondel C, Bostad L, Larsen KK, Hirth A, Vikse BE, Houge G, et al. Agalsidase benefits renal histology in young patients with Fabry disease. J Am Soc Nephrol 2013;24(1): 137-48.

71. Weidemann F, Krämer J, Duning $T$, Lenders $M$, Canaan-Kühl $S$, Krebs $A$, et al. Patients with Fabry disease after enzyme replacement therapy dose reduction versus treatment switch. J Am Soc Nephrol 2014;25(4):837-49.

72. Beck M, Hughes D, Kampmanna C, Pintos-Morell G, Ramaswami U, West M, Giugliani R. Long-term outcomes with agalsidase alfa enzyme replacement therapy: Analysis using deconstructed composite events. Molecular Genetics and Metabolism Reports 14 (2018) 31-35.

73. Eng C, Gurron N, Wilcox WR, Germain DP, Lee P, Waldek S, et al. Safety and efficacy of recombinant human -galactosidase A-replacement therapy in Fabry's disease. N Engl J Med 2001;345:9-16.

74. Skrunes R, Svarstad E, Kampevold Larsen K, Leh S, Tøndel C. Reaccumulation of globotriaosylceramide in podocytes after agalsidase dose reduction in young Fabry patients. Nephrol Dial Transplant 2016;32(5):807-813. 